\title{
Essential nuclear medicine physics
}

\author{
N. C. Purandare, V. Rangarajan \\ Bioimaging Unit, Tata Memorial Hospital, Parel, Mumbai - 400 012, India \\ Correspondence: Dr. V Rangarajan, Bioimaging Unit, Tata Memorial Hospital, Parel, Mumbai - 400 012, India. \\ E-mail: drvrangarajan@gmail.com
}

\section{Second edition \\ Rachel A Powsner and Edward R Powsner Blackwell Publishing 206pp soft bound ISBN 1-4051-0484-8}

This is an excellent, compact yet comprehensive book that elucidates the basic principles of radioactivity and radiation phenomena used in Nuclear Medicine. The book is well-illustrated with appropriate and lucid diagrams. It would be of interest to both the beginner with no prior knowledge of nuclear medicine physics as well as a more experienced reader.

Important topics like interaction of radiation with matter, structure of an atom, radioactivity and scintillation instruments form the initial chapters. While these concepts are discussed in other books of physics, the illustrated approach used here to explain the concepts is outstanding. In addition to radiation detector instruments, the concepts of radiation biology, principles of radiation dosimeter, radiation safety and management of nuclear emergencies are explicitly covered. The topics of SPECT, PET and PET/CT are included. The physics of construction, instrumentation and the principles of raw image generation are explained in a simplified manner. Image reconstruction techniques, attenuation correction are explained in a lucid way.

The extensive illustrations, each of which covers a concept, are self-explanatory. This approach makes the book unique. Objective questions at the end of each topic are another feature of this book that will help those preparing for MCQ based exams. An appendix of worked examples of dose calculations, NRC rules and useful references is included.

The book is ideal for every imaging student for understanding the principles of physics in nuclear medicine. It will also serve as an excellent reference book for the nuclear medicine technologist, physicist and physician.

The book deserves a place on the shelf of every nuclear physician and radiologist and in the library of every institution.

\section{Author Help: Sending a revised article}

1) Include the referees' remarks and point to point clarification to those remarks at the beginning in the revised article file itself. In addition, mark the changes as underlined or coloured text in the article. Please include in a single file

a. referees' comments

b. point to point clarifications on the comments

c. revised article with text highlighting the changes done

2) Include the original comments of the reviewers/editor with point to point reply at the beginning of the article in the 'Article File'. To ensure that the reviewer can assess the revised paper in timely fashion, please reply to the comments of the referees/editors in the following manner.

- $\quad$ There is no data on follow-up of these patients.

Authors' Reply: The follow up of patients have been included in the results section [Page 3, para 2]

- Authors should highlight the relation of complication to duration of diabetes.

Authors' Reply: The complications as seen in our study group has been included in the results section [Page 4, Table] 